\title{
Numerical analysis of temperature distribution of plasma arc with molten pool in plasma arc melting
}

\author{
S.C. Chu, S.S. Lian * \\ Department of Material Science and Engineering, National Taiwan University, Taipei, Taiwan \\ Received 12 December 2003; received in revised form 5 March 2004; accepted 5 March 2004
}

\begin{abstract}
The relationships among the parameters that govern the plasma arc MR (melting and refining) process and the quality of the alloy melts are complicated. Only a simulation of the process can enable the process to be properly controlled and the right compositions to be achieved. The main purpose of this paper is to simulate the temperature distribution in the molten pool using the software FEMLAB. Numerical calculations were based on finite element analysis and the coupling of heat transfer, fluid flow and electromagnetic modules. The results of simulations are verified with the meniscus shape of liquid isothermal line in the solid ingot.
\end{abstract}

(C) 2004 Elsevier B.V. All rights reserved.

Keywords: Plasma arc melting; Simulation; Temperature distribution; FEMLAB

\section{Introduction}

The processing of high quality metal and alloys in modern electronic aerospace and medical engineering industries is difficult using the traditional melting technologies. Therefore, special melting processes such as VAR, VIM, EBM and plasma arc melting are utilized to melt fine alloys, which include titanium alloys, super alloys and electronic target alloys.

Plasma arc melting has some advantages over the other processes in producing reactive and high

\footnotetext{
${ }^{*}$ Corresponding author.

E-mail address: lian@ntu.edu.tw (S.S. Lian).
}

quality alloys. It uses highly concentrated energy, a high arc temperature and fast heat transfer.

It also allows the atmosphere to be controlled, and the required capital investment is reasonable. However, the disadvantage of plasma arc melting is that of the temperature of plasma arc drops dramatically from the center line toward exterior of the arc column comparing to the normal induction heating, hence the heating object will result in inhomogeneous distribution of temperature, which will influence the life of the plasma furnace and the qualities of ingots e.g., segregation and shrinkage etc.

The temperature of molten pool of metals depend on many processing variables such as the magnitude of arc current, the gas flow rate, gas composition, arc length, raw materials, viscosity 
thermal conductivity of gas and heating materials etc.

A experimental approach to find the influence of the processing parameters on the temperature distribution in plasma melting is time-consuming and expensive. Therefore, numerical analyses are efficient to solve these problems. Several studies of heat transfer and velocity field in the molten pool associated with the plasma arc processes have been published [1-5], however few of studies have release the details of simulation methodology and data of systems or equipments. Therefore, this work will try to deal our plasma arc melting with coupled complex modules of heat transfer, electromagnetism and fluid flow by a practical simulation techniques, if the methodology is feasible, then the melting of good qualities of alloys by plasma arc heating in different conditions could be achieved.

Due to the difficulty of measuring the temperature in the plasma arc and molten pool, the verification of the results of simulation will be evaluated indirectly with the shape of molten pool of the liquid metal.

The electromagnetic filed in the arc is used to induce the arc current and the analysis involve the solution of the Maxwell equations to describe the electrical and magnetic field, which are coupled with the Navier-Stokes' equation and heat transfer equation to calculate the velocity fields and temperature distribution of both plasma arc and molten pool.

\section{Governing equation and establishment of model}

In order to save the calculation time and to simplify the analysis, the following assumptions are given in this model:

1. The electromagnetic or Lorentz force is generated by the interaction of induced magnetic field and current density.

2. The momentum of the plasma jet generates a gas drag force applied to the surface of the melting pool.

3. The buoyancy force is generated by the changes of temperature in the melting pool.
4. Plasma fluid exhibits non-compressible laminar flow.

5. It is $2 \mathrm{D}$ axis-symmetric system.

6. Chemical reactions in the molten pool are neglected.

7. The physical properties of the material, such as electrical conductivity, thermal conductivity, entropy, enthalpy and viscosity depend only on temperature.

8. The plasma arc is in a stable state.

9. The working gas is argon.

Governing equation is derived from the Maxwell's equation and Navier-Stokes equation:

(1) Mass conservation:

$\frac{1}{r} \frac{\partial}{\partial r}(\rho r u)+\frac{\partial}{\partial z}(\rho v)=0$

(2) Radial and axial momentum conservation:

$$
\begin{gathered}
\frac{\partial}{\partial t}(\rho u)+\frac{u}{r} \frac{\partial}{\partial r}(\rho r u)-\frac{2}{r} \frac{\partial}{\partial r}\left(\mu r \frac{\partial u}{\partial r}\right)+v \frac{\partial}{\partial z}(\rho u) \\
-\frac{\partial}{\partial z}\left[\mu\left\{\frac{\partial v}{\partial r}+\frac{\partial u}{\partial z}\right\}\right] \\
=-\frac{\partial p}{\partial r}-2 \mu \frac{u}{r^{2}}-j_{z} B_{0} \\
\frac{\partial}{\partial t}(\rho v)+v \frac{\partial}{\partial z}(\rho v)-2 \frac{\partial}{\partial z}\left(\mu \frac{\partial v}{\partial z}\right)+\frac{u}{r} \frac{\partial}{\partial r}(\rho r v) \\
-\frac{1}{r} \frac{\partial}{\partial r}\left[\mu r\left\{\frac{\partial u}{\partial z}+\frac{\partial v}{\partial r}\right\}\right] \\
=-\frac{\partial p}{\partial z}+j_{r} B_{0}-\rho g \beta\left(T=T_{r}\right)
\end{gathered}
$$

(3) Thermal energy conservation:

$$
\begin{aligned}
\frac{\partial}{\partial t}\left(\rho C_{\mathrm{p}} T\right)+\frac{u}{r} \frac{\partial}{\partial r}\left(\rho r C_{\mathrm{p}} T\right)+v \frac{\partial}{\partial z}\left(\rho C_{\mathrm{p}} T\right) \\
=\frac{1}{r} \frac{\partial}{\partial r}\left(\lambda r \frac{\partial T}{\partial r}\right)+\frac{\partial}{\partial z}\left(\lambda \frac{\partial T}{\partial z}\right)-\frac{J_{z}^{2}+J_{\mathrm{r}}^{2}}{\sigma}-S_{\mathrm{R}} \\
\quad+\frac{5}{2} \frac{k}{\mathrm{e}}\left(J_{z} \frac{\partial T}{\partial Z}+J_{\mathrm{r}} \frac{\partial T}{\partial r}\right)
\end{aligned}
$$

(4) Electrical charge conservation:

$\frac{1}{r} \frac{\partial}{\partial r}\left(\sigma r \frac{\partial \phi}{\partial r}\right)+\frac{\partial}{\partial z}\left(\sigma \frac{\partial \phi}{\partial z}\right)=0$

where $B, B_{z}, C_{\mathrm{p}}$, e, $E$ denote magnetic flux vector, azimuthal component for magnetic flux, average 
specific heat of plasma at constant pressure, electronic charge and electric field, respectively.

$J, J_{\mathrm{c}}, J_{\mathrm{r}}, J_{z}, K, P, S_{\mathrm{R}}, T, T_{0}$ stand for current density vector, current density at cathode, radial and axial components of current density, Boltzmann's constant, pressure, radiation heat loss per unit volume, temperature of plasma and ambient temperature.

$u, v, r, z, \mu, \rho, \lambda$ represent axial component of plasma velocity, radial component of plasma velocity, radial coordinate, axial coordinate, molecular viscosity, electrical conductivity of plasma and thermal conductivity of plasma.

\section{The finite element model}

Fig. 1 presents a schematic $2 \mathrm{D}$ axis-symmetry model of a plasma melting system, which includes the plasma gun and plasma arc. Commercial finite element analysis software (FEMLAB), which is a tool box of MATLAB for solving the partial differential equations (PDEs) is used for the simulation work. The Lorentz force induced from the interaction between the electric current and the magnetic fields is calculated in the electromagnetic module as Eq. (i), and then the force is substituted for the electromagnetic force in the flow flux field

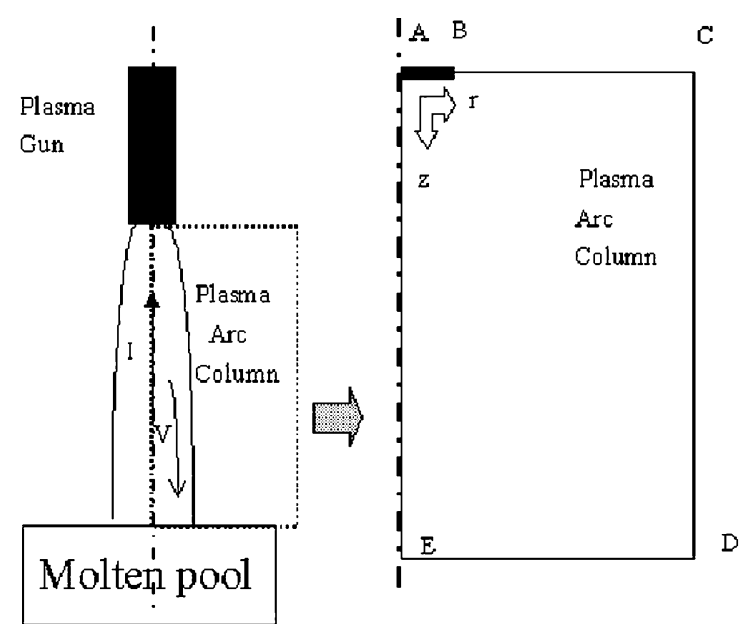

Fig. 1. 2D axis-symmetry model of plasma melting system. to achieve a momentum balance as Eq. (ii). The calculated speed field will be substituted for the flow speed in the heat transfer field. With the heat generated by electrical resistance and electrical flow transports to achieve energy balance as Eq. (iii). A temperature distribution can finally be obtained by repeating this process.

The material properties are obtained using the regression equation with data in Ref. [6]. The finite element analysis uses triangular element with 3086 mesh.

From the electromagnetic field analysis, Maxwell equation and the conservation of electric current are applied to quasi-static plane currents, based on the assumption that the flat surface includes only an electric current.

Considering only the magnetic field perpendicular to the flat surface, the PDE equation is given as follows:

$$
\begin{aligned}
& {\left[\left(H_{z}+M_{z}\right) \frac{\mathrm{d} \mu}{\mathrm{d} H_{z}}+\mu\right] \frac{\partial H_{z}}{\partial t}} \\
& -\nabla \cdot\left(\tilde{\sigma} \nabla H_{z}-\mu v H_{z}-\tilde{\sigma}\left[\begin{array}{c}
-J_{y}^{e} \\
J_{x}^{e}
\end{array}\right]-\mu v M_{z}\right) \\
& =-\mu \frac{\partial M_{z}}{\partial t} \\
& \tilde{\sigma}=\frac{\sigma^{\mathrm{T}}}{\operatorname{det}(\sigma)}
\end{aligned}
$$

In this model, the current density in the plasma gun $\mathrm{AB}$ is $10^{7} \mathrm{Am}^{-2}$ and the electrical potential in the bottom of the boundary DE is zero (Fig. 1); the diameter of the cathode is $4 \mathrm{~mm}$. The magnetic potential is 0 when $t=0$; arc length is $1 \mathrm{~cm}$; relative magnetic conductivity $\left(\mu_{\mathrm{r}}\right)$ is 1 .

Electrical conductivity $(y)$ changes with the temperature $(x)$ according to the following equation [6]:

$$
\begin{aligned}
y= & -2.68 \times 10^{8} X^{3}+6.06 \times 10^{5} \times X^{2}+5.58 \times X \\
& -3960
\end{aligned}
$$

The PDE of 2D mode flow field analysis of incompressible Navier-Stokes equation with additional Lorentz force term is given in Eq. (ii) as follows: 
$\rho \frac{\partial u}{\partial t}-\nabla \cdot \eta\left(\nabla u+(\nabla u)^{\mathrm{T}}\right)+\rho(u \cdot \nabla) u+\nabla p=F$

$\nabla \cdot u=0$

These boundary conditions are setting as follows (Fig. 1):

$\mathrm{AB}: u=0, v=4 \mathrm{~m} / \mathrm{s}$

$\mathrm{BC}: u=v=0$

$\mathrm{CD}: \frac{\partial u}{\partial r}=\frac{\partial v}{\partial r}=0$

$\mathrm{DE}: \frac{\partial u}{\partial z}=\frac{\partial v}{\partial z}=0$

The gas flux density is $3.5 \times 10^{-2} \mathrm{~kg} / \mathrm{m}^{-3}$; the viscosity $(y)$ changes with the temperature $(x)$ as the following equation [6]:

$y=5.467 \times 10^{-12} X^{3}-2.2 \times 10^{-7} X^{2}+0.002543 X$

$$
-6.4
$$

For the part of heat transfer field analysis, the PDE Eq. (iii) has include the term of Joule heat in the energy balance equation, which includes conduction and convection of heat

$\rho C_{\mathrm{p}} \frac{\partial T}{\partial t}+\nabla \cdot\left(-k \nabla T+\rho C_{\mathrm{p}} T_{u}\right)=Q$

The boundary conditions are setting as follows (Fig. 1):
AB: $T=300 \mathrm{~K}$
BC: $T=300 \mathrm{~K}$
$\mathrm{CD}: \frac{\partial T}{\partial r}=0$

At $t=0, T=300 \mathrm{~K}$. $C_{\mathrm{p}}$ is 20.79 .

The dependence of heat conductivity $(y)$ on the temperature $(x)$ is shown in the following equation [6]:

$$
\begin{aligned}
y= & -3.867 \times 10^{-12} X^{3}+1.43 \times 10^{-7} X^{2} \\
& -0.001378 X+4.05
\end{aligned}
$$

The boundary condition on the axis-symmetry centerline (EA) is

$$
\frac{\partial u}{\partial r}=v=\frac{\partial T}{\partial r}=0
$$

\section{Result and discussion}

The results of the simulation are represented in Fig. 2, which displayed the calculated electric field (norm E) with $300 \mathrm{~A}$ arc current. The intensity of the electric field is maximal along the centerline of the electrode and decrease outward in the radial direction. The trend can be considered as reasonable because that the large current will be conduct from the cathode along centerline toward the metal anode in a path of short distance with low arc resistance.

The largest Joule heat and highest temperature will be induced in the same part of arc column. This explanation could be confirmed further from the large velocity field in the same region of arc in Fig. 4.

It is because that a high velocity of ionized gas is driven by a large Lorentz force $(J \times B)$ and the current density is induced in the space of large electric field. Fig. 3 presents the calculated azimuth angle and magnetic field density.

\subsection{Analysis of flow flux field}

Fig. 4 shows the distribution of calculated velocity field. The velocity declines slowly downwards in the radial direction, but is primarily dis-

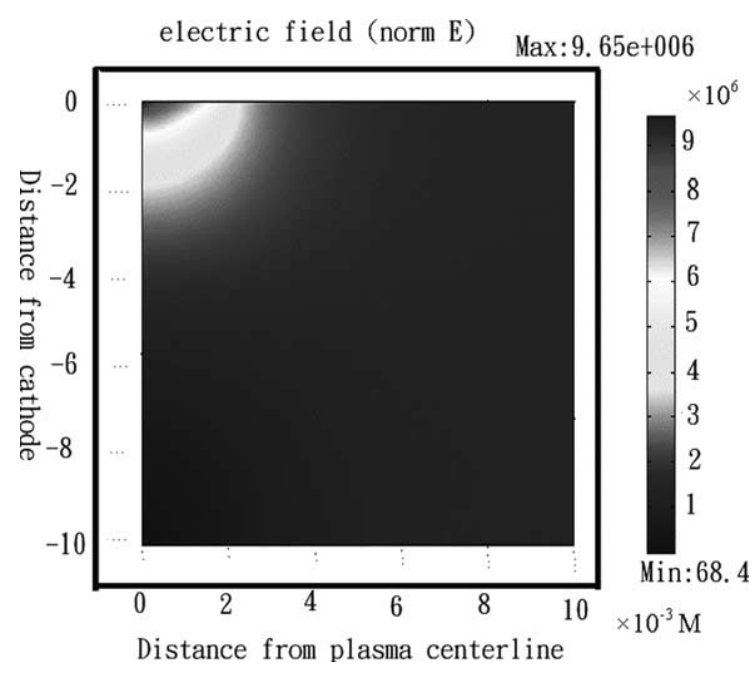

Fig. 2. The calculated electric field with $300 \mathrm{~A}$ arc current. 


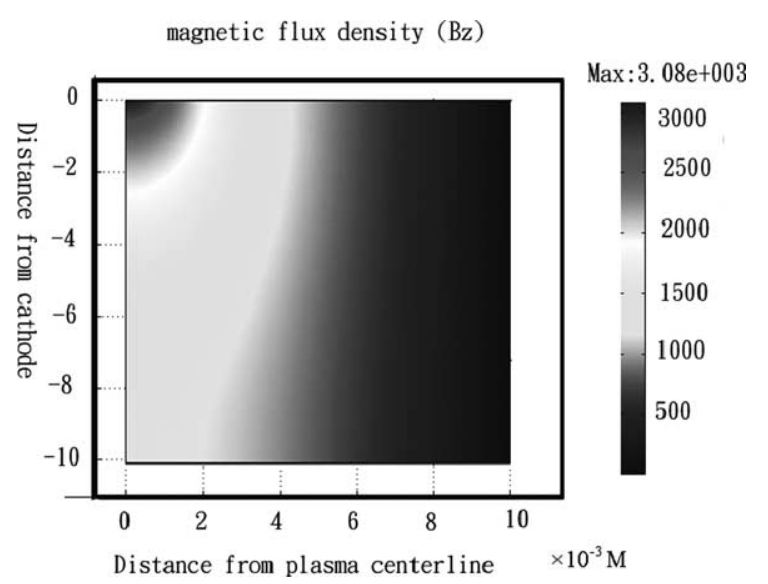

Fig. 3. The azimuth angle magnetic field density.

velocity field

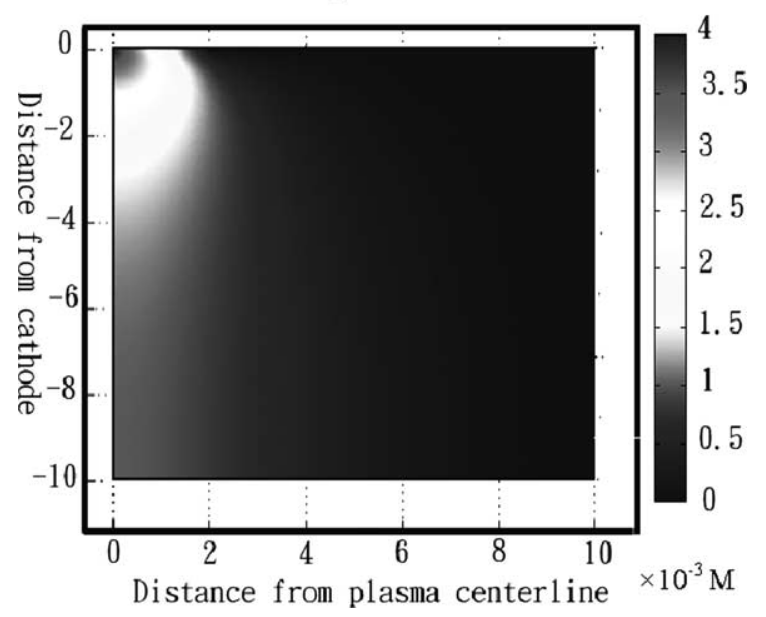

Fig. 4. The calculated velocity field with 300 A arc current.

tributed along the axis. The simulation in this investigation neglected the effect of turbulence.

The results of simulation in this work show similar trend to the report of velocity distribution in Ref. [7].

\subsection{Analysis of temperature}

Fig. 5 plots the temperature distribution of a 500 A current.

The temperature is highest in the center of the plasma column, falling gradually into the sur-

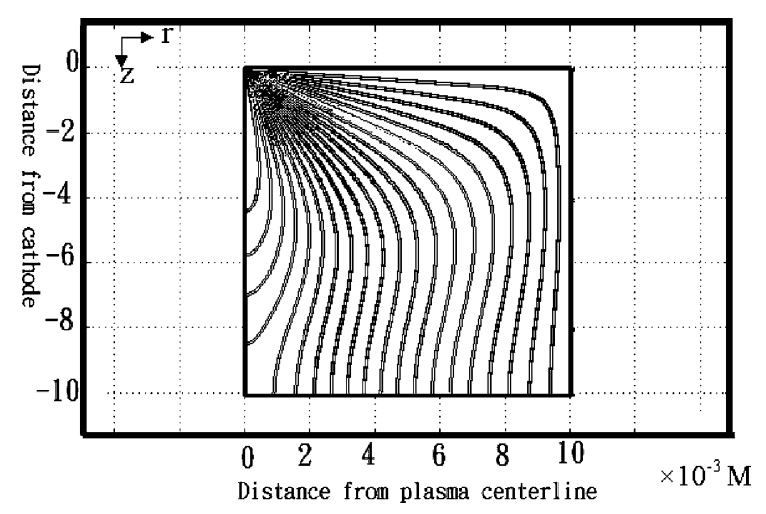

Fig. 5. Temperature isotherm distribution of $500 \mathrm{~A}$ current (isotherm lines from left to right are 21010, 19980, 18940, $17900,16870,15830,14800,13760,12730,11690,10660,9620$ 8584, 7549, 6513, 5478, 4442, 3407, 2371, $1336 \mathrm{~K})$.

roundings. Compare to Fig. 2 the location of high temperature region is corresponding to the high electric filed region along the central line of arc. This could be explained as the consequence of large Joule heating generating in the place of high electric field. Comparing to the result in Ref. [1], which shows a large turning point near the topside, Fig. 5 has more smooth temperature variation. This trend shows similarity to the image of photograph presented in Fig. 6 in our experiment.

The temperature profiles of plasma arc on the interface $1 \mathrm{~cm}$ below electrode and the influence of electric current on the temperature of the plasma arc is shown in Fig. 7. The column of the plasma becomes wider as current is increased. It means

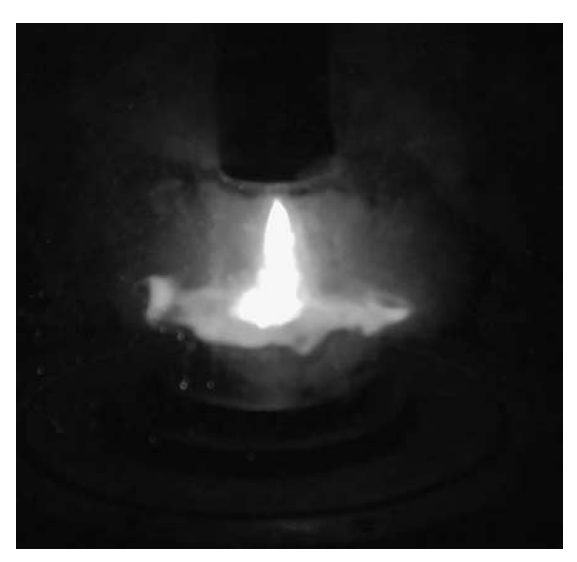

Fig. 6. Photograph of plasma arc in experiment. 


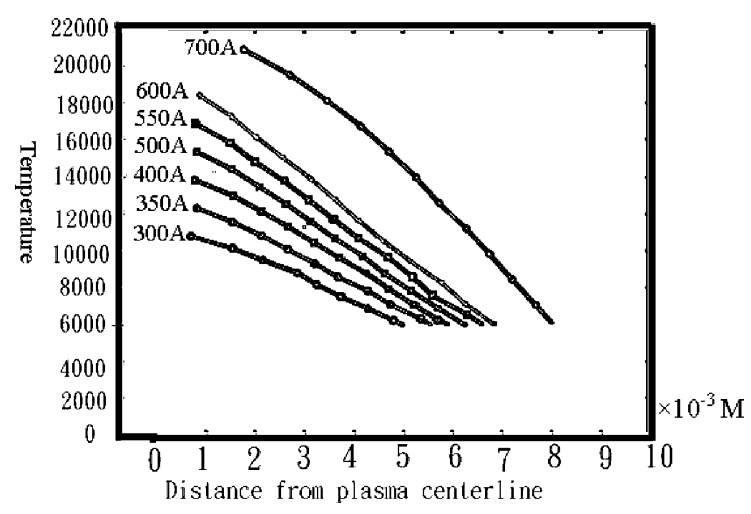

Fig. 7. The temperature profile from center to the edge of crucible below $1 \mathrm{~cm}$ from electrode.

that large current can have a more uniform temperature distribution than the one of small arc current.

In order to calculate the temperature field in metal, a balance of heat and electrical charge were made on the interface between the arc and metal.

Material and thermophysical properties of the molten pool (silicon) [8] are shown below.

Atomic weight of silicon is $28.09(\mathrm{~g} / \mathrm{mol})$, liquid viscosity $\mu_{m p \text {,liq }}$ is $0.94 \times 10^{-3}$ (Pa s) and density $\rho_{m, \text { sol }}$ is $2.34 \times 10^{3}\left(\mathrm{~kg} / \mathrm{m}^{3}\right)$.

Equations are given to express the dependence of the liquid metal density, $\rho_{m, \text { liq }}$, specific heat $\left(\mathrm{kg} / \mathrm{m}^{3}\right)$, thermal conductivity of solid metal, $C p_{m \text {,sol }}(\mathrm{J} / \mathrm{kg} \mathrm{K})$ and $K c_{m, \text { sol }}(\mathrm{W} / \mathrm{m} \mathrm{K})$ to the temperature as follows:

$$
\begin{aligned}
\rho_{m, \text { liq }}= & 2.51 \times 10^{3}-\left(T_{m}-1685\right) \times 0.32 \\
C p_{m, \text { sol }}= & 812.07+13.80 \times 10^{-2} T_{m} \\
& -12.70 \times 10^{6} T_{m}^{-2} \\
K c_{m, \text { sol }}= & 1.08 \times 10^{5} T_{m}^{-1.167}
\end{aligned}
$$

The temperature distributions of calculated heating metal block are shown in Figs. 8 and 9 . The temperature on the interface is different significantly in the side of molten pool and plasma arc. It can be shown that the temperature of $300 \mathrm{~A}$ plasma arc at $1 \mathrm{~cm}$ below the cathode in Fig. 7 is approximately 11,000 , while the temperature of molten pool has 1600 on the same position with $300 \mathrm{~A}$. It is reasonable that the temperature of real molten pool has rarely close to $11,000{ }^{\circ} \mathrm{C}$.

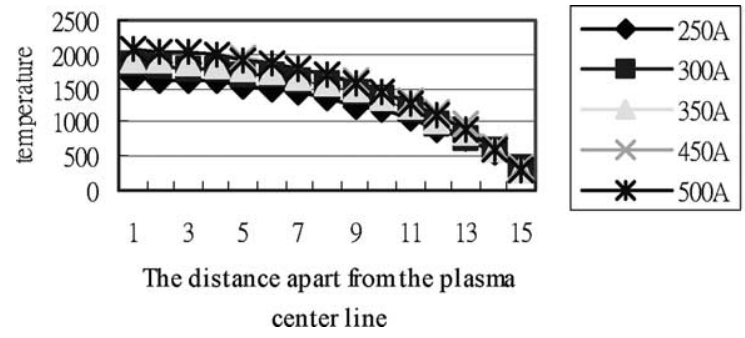

Fig. 8. The result of temperature profile on the surface of molten pool from center to the edge of crucible.

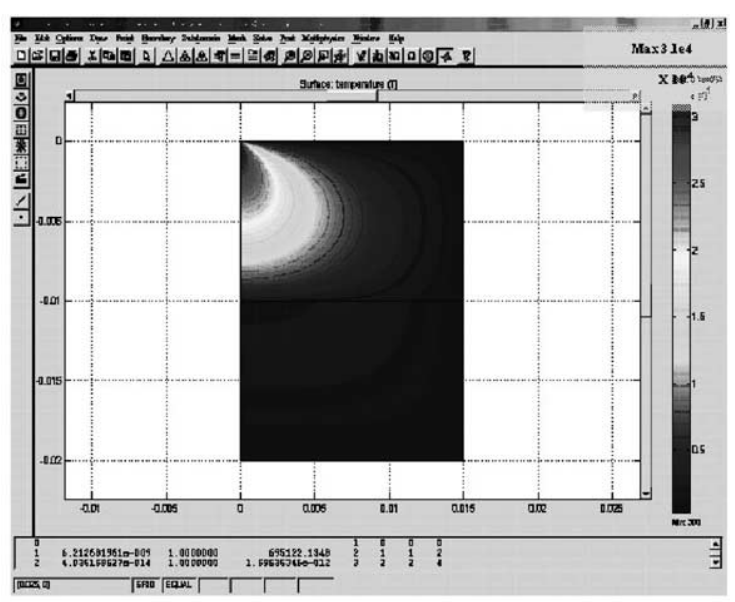

Fig. 9. The temperature distribution in both plasma arc and molten pool.

When the molten pool is present, the plasma arc is direct contacted with the molten pool.

The big difference of temperature between arc and molten pool on the interface is probably because the physical properties differ abruptly between plasma gas and molten pool.

The calculated isotherm in Fig. 9 below the interface has the shape of a meniscus, which is similar to the normal shape of a macro-etched cross-section of a solidified ingot.

\section{Conclusion}

The results of this study have indicated that the methodology of calculation of temperature distribution is feasible. 
The calculated isothermal lines in the molten pool show a meniscus shape. It is similar to the real shape of the liquid in heating metal and an indirect way examination of the feasibility of simulation technique.

The method represents uncomplicated way to simulate complex interactions among different fields in plasma melting.

A larger arc current will induce a more homogenous temperature distribution.

This study also found that the significant difference between the temperature of on side of plasma arc and side of molten liquid.

The calculated temperature in molten pool is reasonable compare to the temperature of real liquid pool in heating metal.

\section{Acknowledgements}

The authors would like to thank the National Science Council of the Republic of China for financially supporting this research under contract no. NSC_91-2216-E-002-033. Thanks will be also given to the Industrial Technology Research Institute Materials Research Laboratories for financially and technical help on this research under contract no. 91-S-A42.

\section{References}

[1] S. Paik, H.D. Nguyen, Numerical modeling of multiphase plasma/soil flow and heat transfer in an electric arc furnace, Int. J. Heat Transfer 38 (7) (1995) 1161-1171.

[2] H. Kurimoto, H.N. Mondal, T. Morisue, Analysis of velocity and temperature fields of molten metal in DC electric ARC furnace, J. Chem. Eng. Jpn. 29 (1) (1996) 7580.

[3] W. Hu, J.D. Lavers, Coupled electro-thermal-flow model for very long electric arcs, IEEE Trans. Magn. 33 (2) (1997) 1726-1729.

[4] S.Y. Kim, B.H. Kang, J.M. Hyun, Forced convection heat transfer from two heated blocks in pulsating channel flow, Int. J. Heat Mass Transfer 41 (3) (1998) 625-634.

[5] A.M. Fudolig, H. Nogami, J.-I. Yagi, ISIJ Int. 36 (9) (1996) 1222-1228.

[6] J. Mostaghimi-Tehrani, E. Pfender, Plasma Chem. Plasma Proces. 4 (2) (1984) 249-256.

[7] J.W. McKelliget, J. Szekely, J. Phys. D: Appl. Phys. 16 (1983) 1007-1022.

[8] A.M. Fudolig, H. Nogami, J.-I. Yagi, Numerical analysis of the flow characteristics and temperature distribution in metal beans subjected to transferred arc plasma impingement, ISIJ 37 (6) (1997) 623-629. 